\title{
Atributos de calidad web para repositorios de datos de investigación en universidades
}

\author{
Alejandro Morales Vargas \\ Universidad de Chile \\ amorales@uchile.cl \\ https://orcid.org/0000-0002-5681-8683
}

\author{
LLUís CodinA \\ Universitat Pompeu Fabra \\ lluis.codina@upf.edu \\ https://orcid.org/0000-0001-7020-1631
}

\section{Website quality attributes for research data repositories in universities}

\begin{abstract}
El nuevo modelo de ciencia abierta requiere de una infraestructura adecuada para preservar y compartir los datos de investigación. Ello ha implicado un desafío tanto para los investigadores como para las universidades y centros. Como respuesta se han creado más de dos mil repositorios de datos en el mundo, un cuarto por parte de estas instituciones, con diferentes softwares especializados. El presente trabajo tiene como objetivo proponer atributos de calidad web para evaluar la interfaz de este tipo de sitios. Para ello se realizó una revisión sistematizada de literatura con directrices internacionales, requisitos técnicos y recomendaciones de expertos, detectando coincidencias. Como resultado se ofrece un protocolo de análisis especialmente

formulado para repositorios de datos de investigación, con parámetros e indicadores sobre la interfaz de usuario, localización, acceso y reutilización, conservación, difusión, evaluación y compromiso institucional. A modo de conclusión se observa la necesidad de atender los aspectos específicos de este tipo de datos, relevando el papel de las bibliotecas universitarias para gestionarlos.
\end{abstract}

RESUMEN ABSTRACT

PALABRAS CLAVE

Repositorios; Datos de investigación; Calidad web; Repositorio de datos; Ciencia abierta; Bibliotecas universitarias
The new Open Science model requires an adequate infrastructure to preserve and share research data. This has involved a challenge for both researchers and universities and centers. In response, more than two thousand data repositories have been created in the world, a quarter by these institutions, with different specialized software. This paper aims to propose web quality attributes to evaluate the interface of this type of sites. For this, a systematic review of literature with international guidelines, technical requirements and expert recommendations was carried out, detecting coincidences. As a result, an analysis protocol specially formulated for repositories of research data is offered, with parameters and indicators on the user interface, location, access and reuse, conservation, dissemination, evaluation and institutional commitment. In conclusion, the need to address the specific aspects of this type of data is noted, highlighting the role of university libraries in managing them.

\section{KEYWORDS}

Repositories; Research data; Website quality; Data repository; Open science; University libraries 


\section{Introducción}

\subsection{Ciencia abierta y datos de investigación}

Nos encontramos frente a una época de cambio en la forma de hacer ciencia, el que implica un desafío tanto para los investigadores como para las universidades y otras instituciones involucradas. Se trata de un nuevo paradigma fundamentado en que la ciencia debe ser abierta, colaborativa y hecha con y para la sociedad (Anglada y Abadal, 2018).

Es la llamada ciencia abierta, que se relaciona con los conceptos de datos abiertos, open peer review, software libre y acceso abierto. Este último se refiere a la práctica de proporcionar el acceso en línea a información científica reutilizable y gratuita. Lo novedoso es que dicha información ya no se expresa solo en artículos de investigación revisados por pares y publicados en revistas académicas, sino también en los propios datos de investigación, ya sea editados o en bruto (European Commission, 2017).

Los datos de investigación se definen como registros de hechos -puntuaciones numéricas, textos, imágenes y sonidos- utilizados como fuentes primarias para la investigación y cada vez son más aceptados en la comunidad científica como necesarios para validar los resultados (OECD, 2007). Ejemplos de estos datos son las estadísticas, mediciones, respuestas a encuestas, grabaciones de entrevistas, fotografías y observaciones resultantes del trabajo de campo.

En concreto, Arano et al. (2011) distinguen como datos primarios a los experimentos científicos; los modelos y simulaciones; las observaciones; los datos derivados, tanto del proceso como de la combinación de datos sin procesar (raw data); los datos referenciales (por ejemplo, estructuras químicas o secuencias genómicas); y el material complementario, como instrucciones de codificación y guías para entrevistadores, entre otros.

Al estar disponibles en formato digital, los usuarios pueden acceder, extraer, explotar, reproducir y difundir datos de investigación de acceso libre y de forma gratuita. Y, por cierto, al estar en abierto pueden reutilizarse también por otros científicos, transformarse mediante nuevos métodos o agregarse a otras fuentes, evitando así la duplicidad de ensayos, dando transparencia a su forma de obtención y permitiendo su validación (Melero y Hernández-San-Miguel, 2014).

Ahora bien, aunque es una tendencia emergente, principalmente en las ciencias naturales, no todas las áreas del conocimiento la han adoptado con la misma extensión. La idiosincrasia particular de los investigadores de ciencias sociales y humanidades podría explicar que pocos de ellos dejen sus datos accesibles (Gómez, Méndez y Hernández-Pérez, 2016).
Esto se puede deber también al desconocimiento de dónde y cómo compartir datos de investigación. La ciencia abierta requiere de una infraestructura tecnológica adecuada (RECODE, 2014) y de un modelo de gestión (OpenAire, 2017). La falta de un marco para organizar, preservar y hacer que los datos estén disponibles a largo plazo, ha resultado en la pérdida o el descarte de valiosos datos de investigación (Witt, 2008).

Es por ello que en los últimos años diversas naciones y organismos internacionales determinantes para la política científica han puesto sus incentivos en el manejo y difusión de los datos. En Estados Unidos, el año 2003 los National Institutes of Health (NIH) instaron a que los investigadores compartiesen las fuentes primarias y otros materiales (Peset, Aleixandre-Benavent, Blasco-Gil y Ferrer-Sapena, 2017). Diez años más tarde, pasó a ser una exigencia por parte de la National Science Foundation (NSF) en sus instrucciones para la financiación de proyectos.

En 2004 los miembros de la Organización para la Cooperación y el Desarrollo Económicos (OECD), junto a China, Rusia y Sudáfrica, aprobaron una declaración sobre el acceso a los datos de investigación generada con fondos públicos (OECD, 2004).

En la misma línea, la comunidad de académicos, bibliotecarios y editores FORCE11 redactó un manifiesto propugnando un cambio en el modelo de creación y el intercambio de conocimientos para mejorar el enlace a los datos (FORCE11, 2011).

Así mismo, universidades e instituciones científicas del Reino Unido han establecido un concordato sobre datos abiertos (UKRI, 2016) que busca garantizar que estén disponibles para su uso por parte de otros miembros de su comunidad de investigación, de acuerdo a los marcos legales, éticos, disciplinarios y regulatorios.

Por su parte, la Unión Europea (UE) promueve activamente hacer públicos los datos de investigación desde la primera convocatoria del programa marco Horizonte 2020. A través de su Open Research Data Pilot obliga a liberar los datos, las publicaciones y sus metadatos tan pronto como sea posible (European Commission, 2017). En la próxima convocatoria 2021-2027 será obligatorio que se confeccione un plan de gestión de datos (Data Management Plan, DMP) y que se depositen los datos en abierto con el fin de incrementar la transparencia y la eficiencia, a través de una rápida diseminación de los resultados y de facilitar su reutilización (Alcalá y Anglada, 2019).

Este depósito de los datos debe hacerse siguiendo los principios FAIR (Findable, Accesible, Interoperable y Reusable), publicados inicialmente como un artículo científico de la revista Scientific Data de Nature, los cuales detallan cualidades precisas y medibles que una publicación de datos debería seguir (Wilkinson et al., 2016). 
En tanto, el Plan S de Science Europe, si bien se refiere a publicaciones, obliga a las revistas a incorporar enlaces a datos y código en repositorios externos (López-Borrull, Ollé-Castellà, Abadal y García-Grimau, 2019)

\subsection{Tipos de repositorios}

Frente a esta necesidad, los repositorios académicos o institucionales -desde la declaración de Budapest (BOAI) considerados espacios de autoarchivo y que representan la denominada vía verde al acceso abierto (Casal Reyes et al., 2013)-, surgieron como una opción natural para la publicación de datos vinculados a la investigación científica.

Ello resulta lógico porque técnicamente son directorios administrados para conservar objetos digitales, con un servidor de documentos y una interfaz en línea que permite a los creadores cargar los datos (Schleußinger y von Rekowski, 2018). En concreto, un repositorio contiene mecanismos para importar, identificar, almacenar, preservar, recuperar y exportar esos objetos digitales (Bustos-González y Fernández Porcel, 2008) y describirlos mediante etiquetas o metadatos que facilitan su recuperación.

Para los científicos los repositorios representan un sitio web que, respondiendo a las especificaciones del autoarchivo, interoperabilidad, acceso libre y gratuito a los contenidos y la preservación a largo plazo, difunde la producción académica de una institución o una disciplina (Casal Vidal, 2018).

En el mundo existen más de 4400 repositorios en acceso abierto según el Registry of Open Access Repositories (ROAR). Más de la mitad son multidisciplinarios y un $45 \%$ está sustentado en el software DSpace, en cifras del Directory of Open Access Repositories (OpenDOAR).

Sin embargo, la mayoría son repositorios de literatura, que no fueron concebidos originalmente para la publicación de conjuntos de datos de investigación (datasets), por lo que requieren de la implementación de adaptaciones técnicas para cumplir con requerimientos como los principios FAIR y otras directrices.

Los repositorios de datos de investigación tienen características particulares. Realizan muchas funciones útiles para la ciencia abierta, siendo claves el almacenamiento de los datasets y el hacer que se puedan descubrir para su posible reutilización (Bugaje y Chowdhury, 2018). Además, entre otras opciones, deben brindar soporte a la gestión de datos de investigación (RDM).

El Registry of Research Data Repositories (Re3data.org) de DataCite consigna más de 2400 registros y, según Kindling et al. (2017), el $24,3 \%$ de los repositorios de datos de investigación del mundo son institucionales. Pero, a diferencia de los de literatura, el $86,2 \%$ son disciplinarios o temáticos (López-Borrull, 2018), siendo este otro de sus elementos distintivos.

Melero y Hernández-San-Miguel (2014) recogen que el panorama internacional presenta repositorios de datos de diferentes tipologías: editados, como por ejemplo Dryad, que cuenta con curaduría de datos; consorciados, como el interuniversitario holandés 4TU.Datacentrum; instrumentales, como Chandra Data Archive, creado en Harvard para imágenes astronómicas; disciplinares, como el indio RKMP, especializado en cultivo de arroz; nacionales, p. e. el Research Data Australia; institucionales, como PURR, de Purdue University (Figura 1); o de propósito general, como Figshare, entre otros. Existen también modelos de pago, como el alemán RADAR (Kraft et al., 2016).

Al comparar repositorios de publicaciones y de conjuntos de datos, es posible constatar que en estos últimos cada dominio de investigación tiene diferentes necesidades, debido principalmente a la diversidad estructural de sus datasets. Los requisitos de metadatos también pueden variar mucho de una disciplina a otra, lo que precisa de modelos suficientemente flexibles (Amorim, Castro, da Silva y Ribeiro, 2015).

Otra diferencia entre estos tipos de repositorios es el manejo de versiones. Para Xie et al. (2016) en los datos las etapas estables son relativamente ambiguas y efímeras. $Y$ a menudo los científicos evitan publicar resultados preliminares, por tanto el repositorio debe ser capaz de tomar instantáneas a la copia de datos de una investigación en progreso. Pero el flujo de trabajo del programa dominante en el mercado (DSpace) no contempla esta opción, por lo que tampoco brinda permisos específicos y opciones de uso compartido.

De ahí que el advenimiento de la ciencia abierta ha representado una encrucijada para las instituciones, debiendo enfrentarse a la decisión de adaptar su repositorio u optar por otras infraestructuras externas multidisciplinares (como Zenodo, desarrollado por el CERN y recomendado por la UE a través del programa OpenAIRE; Mendeley Data; B2Share de EUDAT; o Figshare) o temáticas (GenBank, desarrollado por el NIH para secuencias genéticas del ADN; ArXiv, especializado en matemática y física; Protein Data Bank, para estructuras moleculares 3D; o el National Oceanographic Data Centre, entre otros). Esta situación plantea además nuevos retos de gestión, políticas y almacenamiento, que cada repositorio debe valorar detenidamente (Barrueco Cruz et al., 2017).

\subsection{Papel de las bibliotecas universitarias}

En el caso de las universidades, estas deben desempeñar un rol de liderazgo en su entorno, y para ello deben gestionar el conocimiento como un potencial al servicio de sus objetivos (Bustos-González y Fernández Porcel, 2008). Y encuentran en sus sitios web una herramienta fundamental para la investi- 


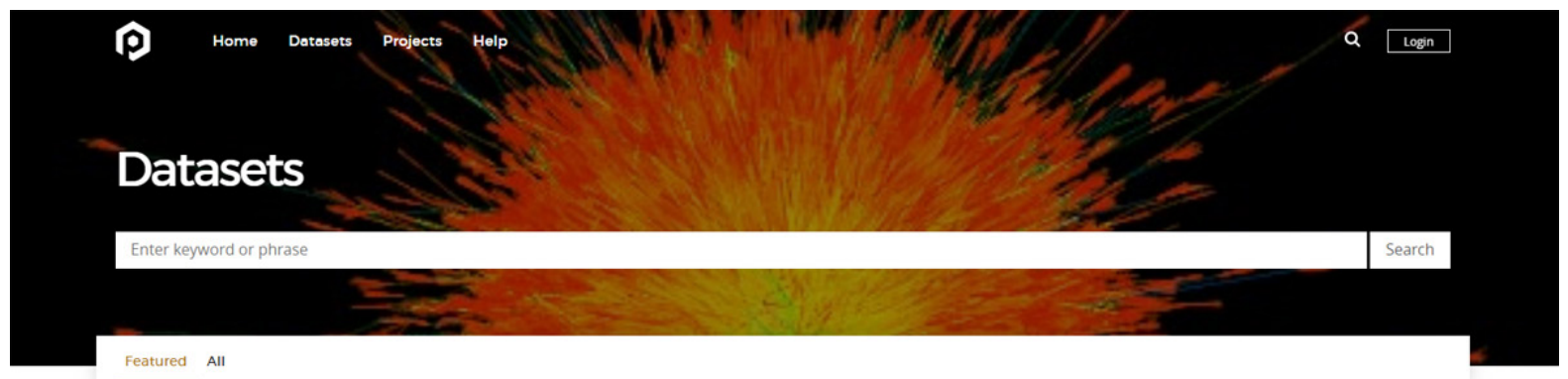

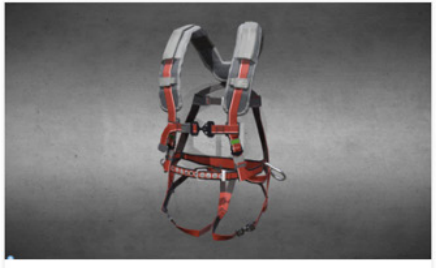

3.D Model of an Harness for OSHA Fall Safety
Training JAMES JENKKNS, JORDYN LUKOMSKI, GEORGE TAKAHASH

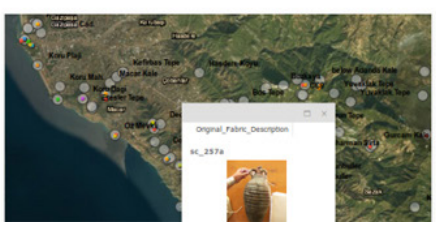

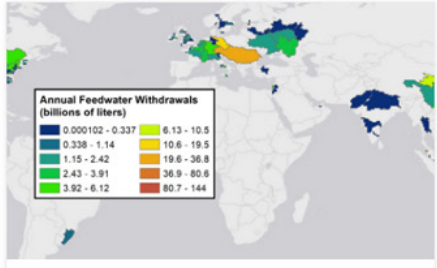

Global Semiconductor Manufacturing Water
Withdrawals KALI D FROST, INEZ HUA

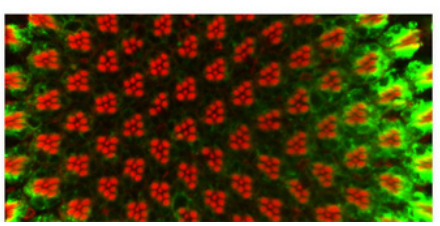

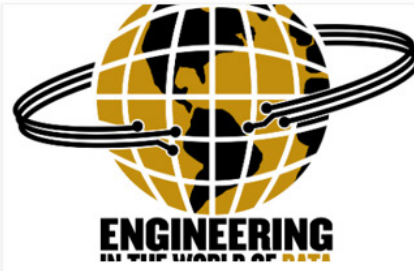

Python Programming Exercise With Specimen Data
for Two Snakes MICHAEL WITT, OLOF OLSSON, SEAN P. BROPHY
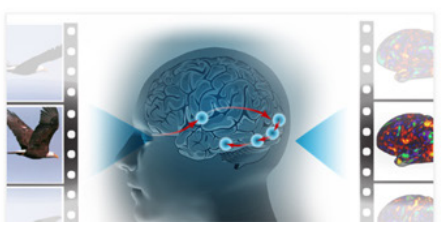

Figura 1. El Purdue University Research Repository (PURR) destaca datasets en su portada.

gación, contribuyendo como ninguna otra a la diseminación e intercambio de contenidos (Orduña-Malea y Aguillo, 2014).

De ahí que la publicación de datos en abierto no solo es un imperativo, sino una oportunidad de extender su quehacer con la comunidad y establecer vínculos con otras instituciones de educación superior, beneficiándose también de la reutilización de los datos de investigación científica (Morales Vargas, 2019).

Sin embargo, la disyuntiva de adaptar su repositorio o publicar los datos en sitios externos no es trivial, pues para las universidades su repositorio institucional juega un papel mucho más estratégico. Este constituye la principal vitrina de su labor científica y una herramienta clave para conseguir una mayor visibilidad y un impacto positivo en los índices de citaciones y en el posicionamiento en los rankings globales (Fernández-Luna, Pérez-Montoro y Guallar, 2019), así como en rankings específicos para repositorios (Aguillo, 2009; Aguillo, Ortega, Fernández y Utrilla, 2010).

Es aquí cuando surge la opción de instalar un segundo repositorio especializado en datos. Sayão y Sales (2016) sostienen que además de indicador de calidad y productividad, un repositorio de datos contribuye a la formación de la memoria científica de las universidades, como registro de las actividades de investigación, complementando a los repositorios institucionales que se centran en publicaciones académicas. Y ello también contribuye a los principios de transparencia universi- taria, que tan demandados son hoy en día.

La experiencia comparada no es concluyente. En el Reino Unido, por ejemplo, cuentan con una red descentralizada de repositorios de datos en cada universidad, mientras que en los Países Bajos disponen de una infraestructura nacional común (Alcalá y Anglada, 2018).

En España, las universidades de la Comunidad de Madrid, agrupadas en el consorcio Madroño, comparten el portal e-cienciaDatos. Lo propio definió crear el Consorci de Serveis Universitaris de Catalunya y por mientras entrega recomendaciones para seleccionar repositorios externos (CSUC, 2016).

La Universitat Pompeu Fabra (UPF), por ejemplo, fue pionera en implementar en su DSpace una comunidad específica denominada «Recursos y datos primarios» (Arano et al., 2011). La Universidad de Chile, en tanto, optó por crear un nuevo repositorio para sus datos de investigación, siendo la primera del país en instalar, traducir y modificar el software Dataverse.

Este último programa informático, impulsado por Harvard University (ver figura 2) y el Institute for Quantitative Social Science, lidera las instalaciones registradas en Re3data.org. Le siguen CKAN, creado por la Open Knowledge Foundation, y desarrollos propios sobre la base de MySQL. Otra alternativa es Invenio, originado en el CERN para almacenar a largo plazo.

En lo que sí hay consenso es en que, aunque los investigadores son en última instancia responsables de la integridad de 


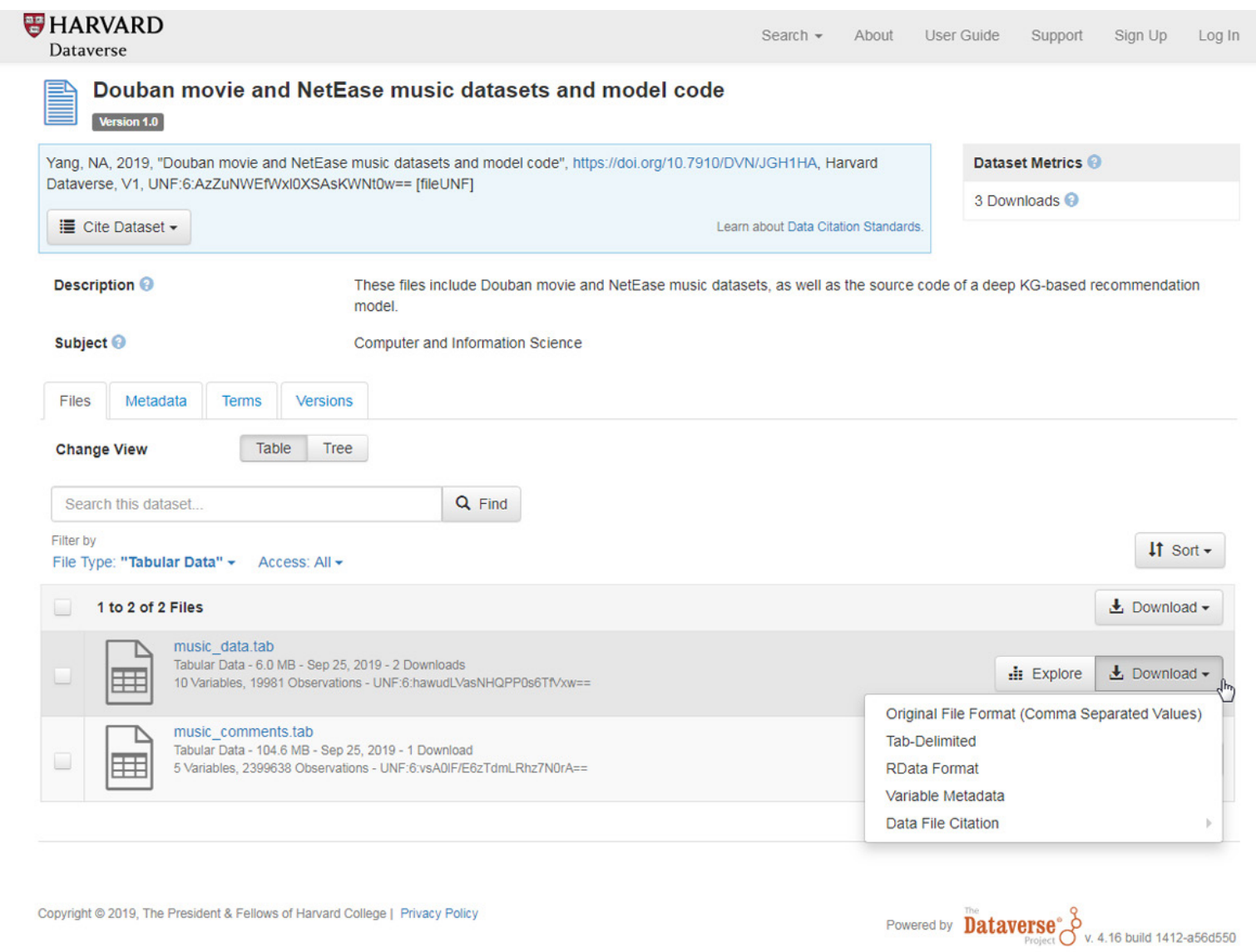

Figura 2. Harvard Datavers brinda opciones de previsualización y exportación de los datos.

los datos en los que se basan sus hallazgos, pocos tienen el conocimiento, el tiempo o los recursos para implementar la gestión de datos o evaluar opciones de almacenamiento de datos en línea (Austin et al., 2015).

Ortúzar (2014) sostiene que las universidades, a través de sus bibliotecas, tienen experiencia promoviendo la publicación y la distribución de datos resultantes de la investigación, así como interconectar recursos científicos, métodos, herramientas y vocabularios. La bibliotecas universitarias, por tanto, están las llamadas a desempeñar un rol activo en la gestión de datos de investigación (Malo de Molina, 2018).

En particular, el perfil profesional del bibliotecario cuenta con la confianza, el espíritu de interdisciplinariedad y colaboración, y la experiencia en la gestión de datos digitales y en su preservación. Por ello, constituyen el personal idóneo para abordar los proyectos de repositorios de datos científicos (Hernández-Pérez y García-Moreno, 2013). Además, los bibliotecarios tienen habilidades para influir en la curación de datos de investigación (Witt, 2008).

Requieren además de la colaboración de especialistas en arquitectura de información y diseño de interfaces centradas en el usuario (Pérez-Montoro, 2010), pues poca atención se ha brindado a la calidad web de los repositorios de datos investigación.

Una revisión simple por este tipo de sitios permite constar que la mayoría cuenta con un bajo nivel de personalización de las opciones que traen los softwares por defecto. Y si ya de por sí los datos suelen ser un terreno árido e intrincado, con mayor razón su interfaz web debería cuidar de ser usable y amigable.

Los estudios sobre la evaluación de la usabilidad de repositorios son un campo casi inexplorado, por lo que se vuelve innovador al incorporar en la metodología de desarrollo y diseño un enfoque centrado en el usuario (Fernández-Luna et al., 2019; González-Pérez, Ramírez-Montoya, García-Peñalvo y Quintas Cruz, 2017).

De ahí que este trabajo se propone generar un sistema de análisis de calidad web especializado (Pedraza-Jiménez, Codina y Guallar, 2016), basado en la evaluación experta, que identifica y define un conjunto de atributos o indicadores a tener en cuenta a la hora de diseñar, implementar y evaluar un repositorio de datos de investigación, en especial, en las universidades. 


\section{Objetivos y metodología}

\subsection{Objetivo general y específicos}

El objetivo general de este trabajo es proponer atributos de calidad web para evaluar la interfaz de usuario de los repositorios de datos de investigación, con énfasis en las necesidades de la comunidad universitaria. A su vez, los objetivos específicos son:

- OE1: Determinar cuáles son las directrices y recomendaciones de expertos con más coincidencias en la literatura sobre este tipo de sitios.

- OEZ: Establecer un conjunto de parámetros e indicadores para evaluar la calidad web de los repositorios de datos de investigación.

- OEZ: Concretar estos atributos en un sistema de análisis de calidad web especializado basado.

\subsection{Metodología}

Para el ejecución del estudio se utilizó el método de la revisión sistematizada de literatura (Codina, 2018; Gough, Oliver, y Thomas, 2017) que permite identificar los principales trabajos de un tema en concreto. Comprende las etapas de búsqueda, evaluación, análisis y síntesis de artículos científicos y publicaciones profesionales.

Se definió «repositorios de datos de investigación» (en inglés, data research repositories) como el campo de estudio y sobre esa base se diseñaron ecuaciones de búsqueda en inglés y español, sin especificar un rango de años determinado. Como universo se utilizaron las más importantes bases de datos académicas multidisciplinarias, Scopus y Web of Science (WoS), en las que se detectaron 144 y 100 documentos, respectivamente.

Esta búsqueda fue complementada con una consulta por el concepto de repositorio institucional -por poseer una interfaz web similar y por ser un tema del que hay mucha más literatura- en combinación con palabras clave relacionadas al análisis, evaluación, directrices, requerimientos y usabilidad.

También se buscó en Google Académico por contener documentación técnica y profesional, y se realizó un mapeo sistemático (Grant y Booth, 2009) a través de las bibliografías de los textos encontrados, alcanzando un total de 284 registros.

Como criterio de evaluación se aplicó la pertinencia temática, excluyendo publicaciones que no entregaban criterios que permitiesen evaluar repositorios. Así se conformó un banco de documentos con 66 publicaciones, las que fueron gestionadas como un grupo en el software de referencias Mendeley ( ${ }^{1}$ ).

Tras su análisis se extrajeron y sistematizaron manualmente listados de factores de evaluación de repositorios, asociados a sus autores y se contabilizó el número de coincidencias para determinar los más importantes e imprescindibles.

Finalmente, estos atributos se organizaron en parámetros e indicadores (Codina, 2008) se muestra un conjunto de parámetros e indicadores de uso general en la evaluación de sitios web, recursos digitales o publicaciones en línea. Como se indica en la primera parte de este documento, no cabe afirmar que sea imprescindible aplicarlos todos en todas las circunstancias, ni tampoco puede afirmarse que sean los únicos necesarios en toda circunstancia. Constituyen sin duda el grueso de los indicadores útiles en casi cualquier escenario, así como proporcionan ideas sobre la clase de aspectos a chequear en un proceso de análisis, pero no tienen vocación de ser exhaustivos ni de ser imprescindibles como un todo. El grado de desarrollo de cada indicador es diferente. Por ejemplo, algunos indicadores se ilustran con ejemplos de buenas y de malas prácticas, mientras que no se hace así en otros. Algunos indicadores se ilustran (literalmente y se articularon tomando como referencia el protocolo para generar sistemas de análisis de medios digitales propuesto por Codina y Pedraza-Jiménez (2016).

\section{Atributos de calidad web}

A continuación, se detallan algunos de los atributos de calidad web más relevantes a tener en cuenta a la hora de desarrollar un repositorio de datos de investigación o de evaluar su interfaz y funciones, junto a requisitos particulares del ámbito universitario.

Estos atributos se organizan en parámetros e indicadores. Cada uno se presenta de este modo: en primer lugar, se propone una definición con referencias bibliográficas, y en segundo lugar se presenta una lista de preguntas de chequeo que pueden ser útiles para facilitar su aplicación o su auditoría mediante una evaluación heurística o experta.

\subsection{Interfaz de usuario}

\subsubsection{Identidad}

Comunicación textual y visual de los rasgos distintivos del repositorio de datos, tales como su objetivo, comunidad destinaria (Westell, 2006), carácter disciplinar, cobertura geográfica (Banzi et al., 2019), afiliación e imagen corporativa (Serrano Vicente, Melero y Abadal, 2014):

- ¿Comunica el nombre del repositorio y su pertenencia institucional? 
- ¿Especifica su propósito, público objetivo, disciplina y país?

- ¿Sigue la identidad gráfica de la universidad a la que pertenece?

\subsubsection{Organización}

Clasificación de los conjuntos de datos por distintos esquemas o estructuras de organización, con el objetivo de ofrecer opciones de acceso a los usuarios y que garanticen un crecimiento ordenado del repositorio (Barrueco Cruz et al., 2017; Bugaje y Chowdhury, 2018; Fernández-Luna et al., 2019; Kim, 2018):

- ¿Clasifica las colecciones bajo un criterio centrado en los usuarios?

- ¿Brinda acceso por área temática o disciplina científica, y no solo por facultad, departamento o centro de la universidad?

- ¿Posibilita la organización de colecciones por proyectos de investigación?

\subsubsection{Etiquetado}

Rotulado textual o icónico para representar las diferentes opciones de navegación y acceso a funcionalidades del repositorio (Aguillo, 2011; Pérez-Montoro, 2010):

- ¿Cuenta cada fichero con un nombre significativo, sin caracteres especiales?

- ¿Indica el formato, extensión y tamaño de cada fichero?

- ¿Consigna la fecha de publicación y versión?

\subsubsection{Navegación}

Estructura arquitectónica que permite la exploración de las diferentes páginas del repositorio (FORCE11, 2011; OpenAire, 2018; Scientific Data, 2019; Whyte, 2015):

- ¿Existe una página propia (landing page) para cada dataset?

- ¿Cada conjunto de datos está enlazado a su publicación original?

- ¿Genera una página de autor que liste los dataset aportados por cada uno?

\subsubsection{Usabilidad}

Facilidad de uso del repositorio, con efectividad, eficiencia y satisfacción por parte de los usuarios (Chaves Café y Kafure Muñoz, 2017; van Wyk y van der Walt, 2017):

- ¿La interfaz es simple, intuitiva y fácil de usar?

- ¿Sigue los estándares y mejores prácticas de diseño web?
- ¿Diferencia claramente los enlaces de navegación y de descarga de ficheros?

\subsubsection{Adaptabilidad}

Capacidad de la interfaz de adecuarse a las necesidades particulares de los usuarios, como la accesibilidad (Rovira, Marcos y Codina, 2007), el multilingüismo (Chaves Café y Kafure Muñoz, 2017), la personalización por roles (Amorim et al., 2015; Bugaje y Chowdhury, 2018) o el diseño adaptativo para móviles (Kim, 2018):

- ¿Cumple con las directrices de accesibilidad WCAG, al menos hasta el nivel AA?

- ¿La interfaz se puede cambiar de idioma, como mínimo del local a inglés?

- ¿Se visualiza correctamente en teléfonos móviles y tabletas?

\subsubsection{Visualización}

Representación gráfica del contenido de los ficheros, ya sea para su selección o explotación (Alcalá y Anglada, 2019; Bugaje y Chowdhury, 2018):

- ¿Se puede previsualizar el contenido de los ficheros antes de descargarlos?

- ¿Facilita el uso de R u otros entornos estadísticos para graficar?

\subsection{Localización}

\subsubsection{Búsqueda}

Indexación y recuperación eficiente de un conjunto de datos en el repositorio, con opciones expansivas (Bach, Schäfer, Enke, Seeger y Gemeinholzer, 2012) o de filtrado (Kramer, 2016) de ayuda a los usuarios:

- ¿Cuenta con búsqueda semántica?

- ¿Es posible aplicar filtros para refinar los resultados (materia, tipo de fichero, año de depósito, etiqueta o palabra clave, colección, autor y afiliación, entre otras)?

- ¿Brinda la opción de búsqueda avanzada?

- ¿Se indica el universo de datasets sobre los que busca?

- ¿La caja de consulta tiene búsqueda elástica, predicción o autocompletado?

\subsubsection{Metadescripción}

Uso de metadatos estándares, con un lenguaje formal y compartido, para representar los datasets (Rousidis, Garoufallou, Balatsoukas y Sicilia, 2014; Wilkinson et al., 2016). Capacidad para soportar múltiples esquemas (Alcalá y Anglada, 2019) 
especializados por área del conocimiento (Austin et al., 2015;

Gómez et al., 2016):

- ¿Utiliza un sistema de metadatos estándar (DataCite, DublinCore u otros)?

- ¿Gestiona diferentes esquemas para disciplinas específicas (p. e. astronomía y astrofísica, ciencias sociales, geoespaciales o de la salud, entre otras)?

- ¿Los metadatos se pueden exportar a otros formatos (DDI, JSON, OpenAire y más)?

- ¿Se utilizan vocabularios controlados (por ejemplo, (OAR)?

\subsubsection{Identificación}

Univocidad y consistencia para designar datasets y autores, mediante el uso de identificadores únicos y persistentes (Aguillo, 2011; Callaghan et al., 2014; OpenAire, 2018; Wilkinson et al., 2016), independiente de su ubicación y número de copias:

- ¿Cuenta asignación automática del identificador único DOI?

- ¿Cada dataset tiene una URL corta y estable en el dominio de la universidad?

- ¿Utiliza ORCID para identificar a los autores?

- ¿Existen identificadores únicos para los proyectos de investigación?

\subsubsection{Citación}

Normalización para una correcta referencia bibliográfica, según las directrices internacionales para citar datos (Alcalá y Anglada, 2019; OpenAire, 2018):

- ¿Proporciona una citación normalizada (DataCite, Data Citation Standard)?

- ¿Facilita la citación en diversos estilos (APA, Vancouver, Chicago, entre otros)?

- ¿Permite exportar la cita a gestores bibliográficos (Mendeley, Zotero, EndNote, Refworks y otros)?

\subsubsection{Descubrimiento}

Posibilidad de encontrar conjuntos de datos relacionados por predicción sobre la base de pertinencia temática y preferencias (Edmunds et al., 2016; RECODE, 2014):

- ¿El sistema recomienda datasets relacionados?

- ¿Se puede explorar por categorías, etiquetas o clústeres de palabras claves?

\subsection{Acceso y reutilización}

\subsubsection{Acceso abierto}

Disponibilidad inmediata - sin requerimientos de registro, suscripción o pago- para descargar los conjuntos de datos de investigación (European Commission, 2016; FORCE11, 2011; OECD, 2007; OpenAire, 2017; Wilkinson et al., 2016):

- ¿Los datos son accesibles de manera abierta, gratuita y universal?

- ¿Se indica el porcentaje total de datasets con acceso abierto?

- ¿Cumple con los principios FAIR?

\subsubsection{Autenticación y embargo}

Registro y validación de usuarios para la gestión del sistema (Kim, 2018; Serrano Vicente, 2017) y control de acceso a datasets que por razones legales o éticas no puedan ser liberados (Alcalá y Anglada, 2019; Amorim et al., 2015; RECODE, 2014):

- ¿Proporciona un inicio de sesión único o se integra con sistemas de autenticación externos (LDAP, Shibboleth u otros)?

- ¿Permite la colaboración de múltiples usuarios para el depósito de datos?

- ¿Cuenta con un flujo con diferentes roles para la gestión de la plataforma?

- ¿Es posible establecer embargo de manera segura sobre datasets sensibles con durante fechas determinadas?

- ¿Brinda la opción de "libro de visitas" para registrar y trazar el uso de ciertos conjuntos de datos?

\subsubsection{Interoperablidad}

Apego a estándares y protocolos que garanticen la correcta comunicación e integración con otros sistemas (Barrueco Cruz et al., 2017; European Commission, 2016; RECODE, 2014; Santos-Hermosa, Ferran-Ferrer y Abadal, 2012):

- ¿Utiliza el protocolo de recogida (harvesting) OAI-PMH?

- ¿Se integra con otros sistemas corporativos de la universidad (CRIS, catálogo, etc.)?

- ¿Fomenta el uso e integración con formatos de fuente abierta o no propietarios?

- ¿Permite intercambio con repositorios de código como GitHub?

\subsubsection{Reutilización}

Fomento y facilidad para la explotación de los datos almacenados (Melero y Hernández-San-Miguel, 2014; Sayão y Sales, 
2016; Serrano Vicente et al., 2014):

- ¿Soporta diferentes licencias de derecho de autor Creative Commons?

- ¿Ofrece una API para la transferencia de datos?

- ¿Permite la exportación a lenguajes XML y JSON?

- ¿Fomenta la inclusión de ficheros con textos explicativos sobre el modo de uso específico de cada dataset (Read me)?

- ¿Facilita la integración herramientas colaborativas?

\subsection{Conservación}

\subsubsection{Almacenamiento}

Capacidad para alojar los conjuntos de datos, soportando el crecimiento de las colecciones (Banzi et al., 2019; Scientific Data, 2019; Uzwyshyn, 2016):

- ¿Admite el archivo de ficheros de hasta $10 \mathrm{~Gb}$ por defecto?

- ¿Considera un almacenamiento incremental, escalar o extensible?

- ¿La transferencia de datos en la ingesta, descarga y exportación es rápida?

- ¿Soporta todo tipo de ficheros y extensiones?

- ¿Dialoga con herramientas de almacenamiento en la nube (Dropbox, Google Drive, Amazon u otros)?

\subsubsection{Preservación}

Política de respaldo que garantice la conservación en el tiempo, seguridad e integridad de los datasets (Alcalá y Anglada, 2019; RECODE, 2014; Sayão y Sales, 2016):

- ¿Considera un período de preservación de al menos 10 años?

- ¿Dispone de dos copias geográficamente distribuidas como mínimo (CLOCKSS)?

- ¿Existen políticas de respaldo, compresión y migración de corto y largo plazo?

- ¿Se garantiza la estabilidad de la plataforma?

- ¿Proporciona herramientas para gestionar la integridad de los datos (replicación, suma de comprobación de datos, entre otros)?

- ¿Cuenta con protocolos de seguridad de la información avanzados?

\subsubsection{Versionado}

Gestión y administración de diferentes versiones de los ficheros del conjunto de datos (Amorim et al., 2015; Kramer, 2016; Santos-Hermosa et al., 2012):

- ¿Permite un control de versiones de los ficheros?
- ¿Mantiene un historial de cambios con registro de fechas?

- ¿Brinda información sobre el estado de los datasets (sin procesar, procesado, curado, publicado)?

\subsection{Difusión}

\subsubsection{Posicionamiento}

Optimización para cautelar la correcta indexación en motores de búsqueda (Bugaje y Chowdhury, 2018; Codina, 2019; Rovira et al., 2007):

- ¿Aplica estrategias SEO?

- ¿Los datos son cosechados por buscadores y recolectores especializados (European Open Science Cloud (EOSC), OpenAIRE, Google DataSearch u otros)?

- ¿Fomenta el posicionamiento por parte de los mismos investigadores (ASEO)?

\subsubsection{Promoción}

Acciones para estimular la visibilidad (Barrueco Cruz et al., 2017; Santos-Hermosa, Ferran-Ferrer y Abadal, 2015; Serrano Vicente, Melero y Abadal, 2017):

- ¿Ofrece la opción de compartir en redes sociales?

- ¿Se integra con redes sociales académicas (Mendeley, Research Gate, Academia, CiteUlike y otras)?

- ¿Permite enviar la URL por correo electrónico?

- ¿Existe suscripción o servicio de alerta?

\subsection{Evaluación}

\subsubsection{Curaduría}

Edición y selección de los datos (Bach et al., 2012; Whyte, 2015; Witt, 2008):

- ¿Permite hacer curación de datos?

- ¿Contempla revisión por pares (peer review)?

- ¿Existe una política para la retirada de datasets?

\subsubsection{Certificación}

Acreditación del repositorio ante organismos de aseguramiento de la calidad (Aguillo, 2008; Aguillo et al., 2010; Callaghan et al., 2014; Texas Digital Library [TDL], 2015):

- ¿Existen políticas explícitas de control de calidad?

- ¿Está registrado en Re3data DataCite, Scientific Data, Databib, Data Citation Index y otros directorios internacionales?

- ¿Cuenta con una certificación (World Data Systems, Core Trust Seal, IASSIST)? 
- ¿Sigue las buenas prácticas del Ranking Webometrics de Repositorios?

\subsubsection{Métrica}

Medición del impacto, presencia web y uso de los conjuntos de datos (Aguillo, 2009, 2018; Bustos-González y Fernández Porcel, 2008):

- ¿Ofrece estadísticas de visita y descarga?

- ¿Consigna métricas alternativas (Altmetric, PlumX)?

- ¿Se realiza trazabilidad del uso de los datasets?

\subsection{Compromiso institucional}

\subsubsection{Respaldo}

Política universitaria de fomento al acceso abierto de sus datos de investigación, dotando al repositorio de gestión, infraestructura tecnológica y sostenibilidad financiera (Banzi et al., 2019; Barrueco Cruz et al., 2017; Edmunds et al., 2016):

- ¿La universidad cuenta con una política de datos de investigación en abierto?

- ¿El repositorio forma parte de las plataformas tecnológicas institucionales?

- ¿Cuenta con dotación de personal bibliotecario dedicado?

- ¿Los costes de mantenimiento y actualización son asumidos por la universidad?

- ¿Existe promoción dentro de la propia universidad y se estimula el depósito de datos de investigación entre sus académicos?

\subsubsection{Asistencia}

Apoyo y soporte a los usuarios para el depósito y gestión de los datos de investigación (Bach et al., 2012; Serrano Vicente et al., 2014):

- ¿Existe un manual de funcionamiento?

- ¿Dispone de guías, tutoriales o preguntas frecuentes?

- ¿Brinda soporte personalizado al usuario a través de mesas de ayuda telefónicas, chats en línea o correo electrónico?

\subsubsection{Aspectos legales}

Condiciones y responsabilidades de la ingesta, intercambio y explotación de los datos de investigación (Barrueco Cruz et al., 2017; OpenAire, 2018; Scientific Data, 2019):

- ¿Hay directrices explícitas que regulen el depósito y uso de los datos?
- ¿Existe un contrato, mandato o acuerdo formal para ser firmado por el generador de datos y el repositorio especificando los roles y responsabilidades de cada uno?

- ¿El autor reconoce al depositar que no está infringiendo ningún derecho de propiedad intelectual?

- ¿Se cautela el cumplimiento de la normativa vigente?

\section{Conclusiones}

Los repositorios surgen como la plataforma natural para la conservación de los datos de investigación, cada vez más importantes en el contexto de la ciencia abierta. Especializados o multidisciplinarios, estos sitios web también cuentan con las características apropiadas para la difusión y para estimular la reutilización de las fuentes primarias de la labor científica.

Los datos de investigación contienen un enorme valor estratégico para las universidades, las que no deben delegar la tarea de su adecuado resguardo, ya sea adaptando sus repositorios institucionales de literatura o desarrollando nuevos espacios exclusivamente para los conjuntos de datos.

Las bibliotecas universitarias cuentan con vasta experiencia en la gestión de información científica y sin duda sus profesionales son los llamados a liderar la implementación y gestionar estos repositorios.

La mayoría de los autores y directrices internacionales coinciden en los requisitos mínimos que este tipo de sitios deberían cumplir. El acceso abierto, el uso de identificadores únicos y persistentes, la interoperabilidad y el apego a estándares, la posibilidad de establecer embargos, la capacidad de almacenamiento, los esquemas de metadatos diferenciados por disciplinas, las métricas de uso y la curación de datos, son los que más menciones alcanzan.

También destacan atributos de calidad web orientados a mejorar la experiencia de usuario de su interfaz, aspecto poco explorado pero no por ello menos importante. La clasificación facetada, las opciones de exportación, el correcto etiquetado, los filtros de búsqueda, la facilidad de uso, el enlazado al artículo original y la existencia de páginas propias por cada dataset, son algunas de las con mayor número de coincidencias.

Estas características fueron organizadas en parámetros e indicadores con preguntas de chequeo rápido para ayudar a los responsables de la biblioteca a elegir el software de gestión adecuado o evaluar el repositorio ya implementado. Incluso pueden servir como elemento de decisión a los propios investigadores a la hora de diseñar y cumplir con sus planes de gestión de datos.

Y aunque no son condiciones inherentes al repositorio en tanto sitio web, este trabajo ha querido incorporar también las 
dimensiones de evaluación y compromiso institucional porque sin duda condicionan su éxito. Las universidades que han explicitado su política de acceso abierto deben traducirlo en acciones concretas que garanticen la adecuada gestión y sostenibilidad de los repositorios, en concordancia con su misión de generar y transmitir conocimiento.

\section{Agradecimientos}

A Gabriela Ortúzar y Rodrigo Donoso Vegas de la Dirección de Servicios de Información y Bibliotecas (SISIB) de la Universidad de Chile por su colaboración e información.

Este trabajo cuenta con el apoyo de la Comisión Nacional Científica y Tecnológica (Conicyt) de Chile, a través del programa Becas Chile para Doctorado en el Extranjero.

\section{Notas}

Bibliografía disponible en el grupo público de Mendeley Data research repositories: https://www.mendeley.com/community/data-research-repositories/

\section{Referencias}

Aguillo, I. F. (2009). Métrica de repositorios y evaluación de la investigación. Anuario ThinkEPI, 3, 40-41.

Aguillo, I. F. (2011). Building web indicators for the EU OA repository. En Workshop on New Research Lines in Informetrics. Madrid: IPP-CCHS (CSIC).

Aguillo, I. F. (2018). Altmetrics of the Open Access Institutional Repositories: A Webometrics Approach. En STI 2018 Conference Proceedings (pp. 159-169). Leiden: Centre for Science and Technology Studies (CWTS).

Aguillo, I. F., Ortega, J. L., Fernández, M., y Utrilla, A. M. (2010). Indicators for a webometric ranking of open access repositories. Scientometrics, 82(3), 477-486. https://doi.org/10.1007/s11192-010-0183-y

Alcalá, M., y Anglada, L. (2018). Gestió de dades de recerca en les biblioteques de les Universitats de Catalunya. En 15es Jornades Catalanes d'Informació i Documentació (p. 12). Barcelona: Àrea de Ciència Oberta, Consorci de Serveis Universitaris de Catalunya (CSUC).

Alcalá, M., y Anglada, L. (2019). FAIR x FAIR: Requisitos factibles, alcanzables e implementables para un repositorio de datos de investigación FAIR (Consorci de Serveis Universitaris de Catalunya, CSUC).

Amorim, R. C., Castro, J. A., da Silva, J. R., y Ribeiro, C. (2015). A Comparative Study of Platforms for Research Data Management: Interoperability, Metadata Capabilities and Integration Potential. En A. Rocha, A. M. Correia, S. Costanzo, y L. P. Reis (Eds.), New Contributions in Information Systems and Technologies. Advances in Intelligent Systems and Computing (pp. 101-111). Springer, Cham. http://doi. org/10.1007/978-3-319-16486-1_10

Anglada, L., y Abadal, E. (2018). ¿Qué es la ciencia abierta? Anuario ThinkEPI, 12(0), 292. http://doi.org/10.3145/thinkepi.2018.43

Arano, S., Martínez, G., Losada, M., Villegas, M., Casaldàliga, A., y Bel, N. (2011). La comunidad «Recursos y datos primarios» de la
Universitat Pompeu Fabra: los repositorios institucionales como infraestructuras científicas: estudio de caso. Revista Española de Documentación Científica, 34(3), 385-407. http://doi.org/10.3989/ redc.2011.3.834

Austin, C. C., Brown, S., Fong, N., Humphrey, C., Leahey, A., y Webster, P. (2015). Research Data Repositories: Review of Current Features, Gap Analysis, and Recommendations for Minimum Requirements. IASSIST Quarterly, 39(4), 24-38.

Bach, K., Schäfer, D., Enke, N., Seeger, B., y Gemeinholzer, B. (2012). A comparative evaluation of technical solutions for long-term data repositories in integrative biodiversity research. Ecological Informatics, 11, 16-24. http://doi.org/10.1016/J.ECOINF.2011.11.008

Banzi, R., Canham, S., Kuchinke, W., Krleza-Jeric, K., Demotes-Mainard, J., y Ohmann, C. (2019). Evaluation of repositories for sharing individual-participant data from clinical studies. Trials, 20(1), 169. https:// doi.org/10.1186/s13063-019-3253-3

Barrueco Cruz, J. M., Andrés Rodríguez, A., Rico Castro, P., Coslado Bernabé, M. Á., Azorín Millaruelo, C., Bernal, I., ... Prats Prat, J. (2017). Guía para la evaluación de repositorios institucionales de investigación. FECYT, RECOLECTA, CRUE y REBIUN.

Bugaje, M., y Chowdhury, G. (2018). Identifying Design Requirements of a User-Centered Research Data Management System. En M. Dobreva, A. Hinze, y M. Žumer (Eds.), Maturity and Innovation in Digital Libraries. ICADL 2018 (pp. 335-347). Cham: Springer International Publishing. http://doi.org/10.1007/978-3-030-04257-8_35

Bustos-González, A., y Fernández Porcel, A. (2008). Directrices para la creación de repositorios institucionales en universidades y organizaciones de educación superior.

Callaghan, S., Tedds, J., Kunze, J., Khodiyar, V., Lawrence, R., Mayernik, M. S., ... Whyte, A. (2014). Guidelines on Recommending Data Repositories as Partners in Publishing Research Data. International Journal of Digital Curation, 9(1), 152-163. https://doi.org/10.2218/ijdc.v9i1.309

Casal Reyes, M., Borgoños Martínez, M. D., Casaldàliga, A., Gómez Castaño, J., Guijarro, C., Ortiz Uceta, E., ... Terroba Pascual, I. (2013). El acceso abierto en las universidades españolas: estado de la cuestión y propuestas de mejora. Métodos de informacion, 4(6), 55-90. https:// doi.org/10.5557/IIMEI4-N6-055090

Casal Vidal, S. (2018, junio 24). Evaluación de repositorios institucionales y bibliotecas digitales de centros no universitarios en España. Universidad Carlos III de Madrid.

Chaves Café, L., y Kafure Muñoz, I. (2017). Evaluación de la Usabilidad del Repositorio Institucional de la Universidad de Brasília. Revista General de Información y Documentación, 27(1), 87-106. https://doi. org/10.5209/RGID.56563

Codina, L. (2008). Sistema general de análisis y evaluación de sitios web: parámetros e indicadores. Metodología de análisis y evaluación de recursos digitales en línea (Vol. 1). Barcelona: Universitat Pompeu Fabra.

Codina, L. (2018). Revisiones bibliográficas sistematizadas: procedimientos generales y framework para ciencias humanas y sociales. Barcelona: Departamento de Comunicación, Universitat Pompeu Fabra.

Codina, L. (2019). SEO académico: significación, componentes y fases. En EDICIC 2019. Barcelona.

Codina, L., y Pedraza-Jiménez, R. (2016). Características y componentes de un sistema de análisis de medios digitales el SAAMD. En R. Pedraza-Jiménez, L. Codina, y J. Guallar (Eds.), Calidad en sitios web: 
Método de análisis general, e-commerce, imágenes, hemerotecas y turismo (pp. 15-39). Barcelona: Editorial UOC.

CSUC. (2016). Recomanacions per seleccionar un repositori per al dipòsit de dades de recerca. RECERCAT (Dipòsit de la Recerca de Catalunya).

Edmunds, R., L'Hours, H., Rickards, L., Trilsbeek, P., Vardigan, M., y Mokrane, M. (2016). Core Trustworthy Data Repositories Requirements.

European Commission. (2016). Guidelines on FAIR Data Management in Horizon 2020. Recuperado de https://ec.europa.eu/research/participants/data/ref/h2020/grants_manual/hi/oa_pilot/h2020-hi-oadata-mgt_en.pdf

European Commission. (2017). Guidelines to the Rules on Open Access to Scientific Publications and Open Access to Research Data in Horizon 2020. Recuperado de https://www.openuphub.eu/52-openaccess/training-material/59-guidelines-to-the-rules-on-open-access-to-scientific-publications-and-open-access-to-research-datain-horizon-2020

Fernández-Luna, A., Pérez-Montoro, M., y Guallar, J. (2019). Metodología para la mejora arquitectónica de repositorios universitarios. Anales de Documentación, 22(2). https://doi.org/10.6018/ ANALESDOC.356431

FORCE11. (2011). FORCE11 Manifiesto: Improving Future Research Communication and e-Scholarship. Recuperado de https://www. force11.org/about/manifesto

Gómez, N. D., Méndez, E., y Hernández-Pérez, T. (2016). Datos y metadatos de investigación en ciencias sociales y humanidades: una aproximación desde los repositorios temáticos de datos. El Profesional de la Información, 25(4), 545-555. https://doi.org/10.3145/epi.2016.jul.04

González-Pérez, L. I., Ramírez-Montoya, M. S., García-Peñalvo, F. J., y Quintas Cruz, J. E. (2017). Usability evaluation focused on user experience of repositories related to energy sustainability: A Literature Mapping. En Proceedings of the 5th International Conference on Technological Ecosystems for Enhancing Multiculturality - TEEM 2017 (pp. 1-11). New York, New York, USA: ACM Press. https://doi. org/10.1145/3144826.3145385

Gough, D., Oliver, S., y Thomas, J. (2017). An introduction to systematic reviews (2nd edition). London: SAGE Publications Ltd.

Grant, M. J., y Booth, A. (2009). A typology of reviews: an analysis of 14 review types and associated methodologies. Health Information y Libraries Journal, 26(2), 91-108. https://doi.org/10.1111/j.14711842.2009.00848.x

Hernández-Pérez, T., y García-Moreno, M.-A. (2013). Datos abiertos y repositorios de datos: nuevo reto para los bibliotecarios. El Profesional de la Información, 22(3), 259-263. https://doi.org/10.3145/ epi.2013.may.10

Kim, S. (2018). Functional Requirements for Research Data Repositories. International Journal of Knowledge Content Development y Technology, 8(1), 25-36. https://doi.org/10.5865/IJKCT.2018.8.1.025

Kindling, M., Pampel, H., van de Sandt, S., Rücknagel, J., Vierkant, P., Kloska, G., ... Scholze, F. (2017). The Landscape of Research Data Repositories in 2015: A re3data Analysis. D-Lib Magazine, 23(3/4). https://doi.org/10.1045/march2017-kindling

Kraft, A., Razum, M., Potthoff, J., Porzel, A., Engel, T., Lange, F., ... Furtado, F. (2016). The RADAR Project-A Service for Research Data Archival and Publication. ISPRS International Journal of Geo-Information, 5(3), 28. https://doi.org/10.3390/ijgi5030028
Kramer, S. (2016). Matrix of use cases and functional requirements for research data repository platforms. Repository Platforms for Research Data Interest Group.

López-Borrull, A. (2018). Evolución de repositorios temáticos y megarrevistas: visión 2018. Anuario ThinkEPI, 12, 316. https://doi. org/10.3145/thinkepi.2018.47

López-Borrull, A., Ollé-Castellà, C., Abadal, E., y García-Grimau, F. (2019). Plan S: oportunidades y amenazas para las revistas de Humanidades y Ciencias Sociales. En E. P. de la I. y F. Dialnet (Ed.), $9^{a}$ Conferencia internacional sobre revistas de Ciencias Sociales y Humanidades (pp. 1-22). Logroño: El Profesional de la Información y Fundación Dialnet.

Malo de Molina, T. (2018). De datos y otros demonios: nuevos retos de la biblioteca universitaria en la investigación. En Consorcio Madroño (Ed.), Jornada Madroño/InvestigaM de buenas prácticas en el apoyo a la investigación. Madrid: Universidad Carlos III de Madrid.

Melero, R., y Hernández-San-Miguel, J. (2014). Acceso abierto a los datos de investigación, una vía hacia la colaboración científica. Revista Española de Documentación Científica, 37(4), e066. https:// doi.org/10.3989/redc.2014.4.1154

Morales Vargas, A. (2019). Datos abiertos y visualización de información en sitios web de universidades chilenas: una asignatura pendiente. En L. Brossi y T. Dodds (Eds.), Visualización de datos: Periodismo y Comunicación en la era de la información visual (pp. 15-26). Santiago de Chile: Editorial Universitaria.

OECD. (2004). Declaration on Access to Research Data from Public Funding. Paris. Recuperado de https://legalinstruments.oecd.org/en/ instruments/157

OECD. (2007). OECD Principles and Guidelines for Access to Research Data from Public Funding. Paris. Recuperado de https://www.oecd. org/sti/inno/38500813.pdf

OpenAire. (2017). Research data management. Understanding research data management. Briefing paper. Recuperado de https:// www.openaire.eu/briefpaper-rdm-infonoads/view-document

OpenAire. (2018). How to select a data repository? Recuperado de https://www.openaire.eu/opendatapilot-repository-guide

Orduña-Malea, E., y Aguillo, I. F. (2014). Cibermetría: midiendo el espacio red. Barcelona: UOC.

Ortúzar, G. (2014). Publicación digital en las universidades y el nuevo papel de las bibliotecas. Anales de la Universidad de Chile, (6), 175-186. https://doi.org/10.5354/0717-8883.2014.31831

Pedraza-Jiménez, R., Codina, L., y Guallar, J. (2016). Calidad en sitios web: Método de análisis general, e-commerce, imágenes, hemerotecas y turismo. Barcelona: Editorial UOC.

Pérez-Montoro, M. (2010). Arquitectura de la información en entornos web. Gijón: Ediciones Trea.

Peset, F., Aleixandre-Benavent, R., Blasco-Gil, Y., y Ferrer-Sapena, A. (2017). Datos abiertos de investigación. Camino recorrido y cuestiones pendientes. Anales de Documentación, 20(1). https://doi. org/10.6018/analesdoc.20.1.272101

RECODE. (2014). Infrastructure and technology challenges (Policy RECommendations for Open Access to Research Data in Europe). London.

Rousidis, D., Garoufallou, E., Balatsoukas, P., y Sicilia, M.-A. (2014). Data Quality Issues and Content Analysis for Research Data Reposi- 
tories: The Case of Dryad. https://dx.doi.org/10.3233/978-1-61499409-1-49

Rovira, C., Marcos, M.-C., y Codina, L. (2007). Repositorios de publicaciones digitales de libre acceso en Europa: análisis y valoración de la accesibilidad, posicionamiento web y calidad del código. El Profesional de la Información, 16(1), 24. https://doi.org/10.3145/epi.2007. ene.03

Santos-Hermosa, G., Ferran-Ferrer, N., y Abadal, E. (2012). Recursos educativos abiertos: repositorios y uso. El Profesional de la Información, 21(2), 136-145. https://doi.org/10.3145/epi.2012.mar.03

Santos-Hermosa, G., Ferran-Ferrer, N., y Abadal, E. (2015). Evaluación de repositorios educativos. En XIV Workshop REBIUN sobre proyectos digitales. VI Jornadas OS-Repositorios (p. 14). Córdoba: REBIUN.

Sayão, L. F., y Sales, L. F. (2016). Algumas considerações sobre os repositórios digitais de dados de pesquisa. Informação y Informação, 21(2), 90. https://doi.org/10.5433/1981-8920.2016v21n2p90

Schleußinger, M., y von Rekowski, T. (2018). Research Data Repositories: Overview of the properties, functions, and types of repositories (FoDaKo project - Forschungsgemeinschaft in Kooperation).

Scientific Data. (2019). Recommended Data Repositories. Recuperado 29 de agosto de 2019, a partir de https://www.nature.com/sdata/ policies/repositories

Serrano Vicente, R. (2017). Evaluación de los repositorios institucionales de acceso abierto en España. (Tesis Doctoral). Universitat de Barcelona. Recuperado de https://www.tdx.cat/handle/10803/463047\#page $=1$

Serrano Vicente, R., Melero, R., y Abadal, E. (2014). Indicadores para la evaluación de repositorios institucionales de acceso abierto. Anales de Documentación, 17(2). https://doi.org/10.6018/ analesdoc.17.2.190821

Serrano Vicente, R., Melero, R., y Abadal, E. (2017). Evaluación de repositorios de acceso abierto de instituciones de investigación españolas. Propuestas para el éxito. En ECA 2017 Ecosistemas del Conocimiento Abierto. Salamanca.

Texas Digital Library [TDL]. (2015). TDL Data Management Working Group Report.

UKRI. (2016). Concordat on Open Research Data. Recuperado de https://www.ukri.org/files/legacy/documents/concordatonopenresearchdata-pdf

Uzwyshyn, R. (2016). Research Data Repositories: The What, When, Why and How. Computers in Libraries, 2016., 36(3).

van Wyk, J., y van der Walt, I. (2017). Criteria and evaluation of research data repository platforms at the University of Pretoria, South Africa. En eResearch Africa 2017. Cape Town: University of Pretoria. http://doi.org/10.13140/RG.2.2.13736.47366

Westell, M. (2006). Institutional repositories: proposed indicators of success. Library Hi Tech, 24(2), 211-226. https://doi. org/10.1108/07378830610669583

Whyte, A. (2015). Where to keep research data: DCC Checklist for Evaluating Data Repositories. Edinburgh: Digital Curation Centre.

Wilkinson, M. D., Dumontier, M., Aalbersberg, Ij. J., Appleton, G., Axton, M., Baak, A., ... Mons, B. (2016). The FAIR Guiding Principles for scientific data management and stewardship. Scientific Data, 3(1), 160018. https://doi.org/10.1038/sdata.2016.18

Witt, M. (2008). Institutional Repositories and Research Data Cura- tion in a Distributed Environment. Library Trends, 57(2), 191-201. https://doi.org/10.1353/lib.0.0029

Xie, Z., Speer, J., Chen, Y., Jiang, T., Brittle, C., y Mather, P. (2016). Developing Institutional Research Data Repository: A Case Study. https:// doi.org/10.1007/978-3-319-49304-6_7

\section{CV}

Alejandro Morales Vargas. Investigador del Programa de Doctorado del Departamento de Comunicación de la Universitat Pompeu Fabra (UPF) y académico del Instituto de la Comunicación e Imagen (ICEI) de la Universidad de Chile. Colaborador del Grupo de Investigación de Documentación Digital y Comunicación Interactiva (DigiDoc) de la UPF. Periodista y Licenciado en Comunicación Social por la Universidad de Chile; Máster en Gestión de Contenidos Digitales por la Universitat de Barcelona. Se ha desempeñado como editor de sitios web, profesor universitario en grado y postgrado, evaluador de proyectos y consultor en arquitectura de información. Fue creador del Diplomado en Periodismo Digital y Gestión de Medios en Internet del ICEI y jefe de Medios Digitales en la Dirección de Servicios de Información y Bibliotecas (SISIB), ambos en la U. de Chile. http://uchile.cl/c46659

Lluís Codina. Doctor en Ciencias de la Comunicación por la Universitat Autònoma de Barcelona. Profesor titular del Departamento de Comunicación de la Universitat Pompeu Fabra (UPF). Coordinador del Grupo de Investigación en Documentación Digital y Comunicación Interactiva (DigiDoc). Coordinador del Master Universitario en Comunicación Social (MUCS). Docente de la Facultad de Comunicación, en los Grados de Periodismo y de Comunicación Audiovisual. Docente de los Masters Universitarios Online en Documentación Digital y en Buscadores de la Barcelona School of Management de la UPF. Codirector del Observatorio de Cibermedios. Coinvestigador principal del proyecto «Creación y contenido interactivo en la comunicación de información audiovisual: audiencias, diseño, sistemas y formatos». CS02015-64955-C4-2-R (MINECO/ FEDER), Ministerio de Economía y Competitividad, España. http://www.lluiscodina.com 


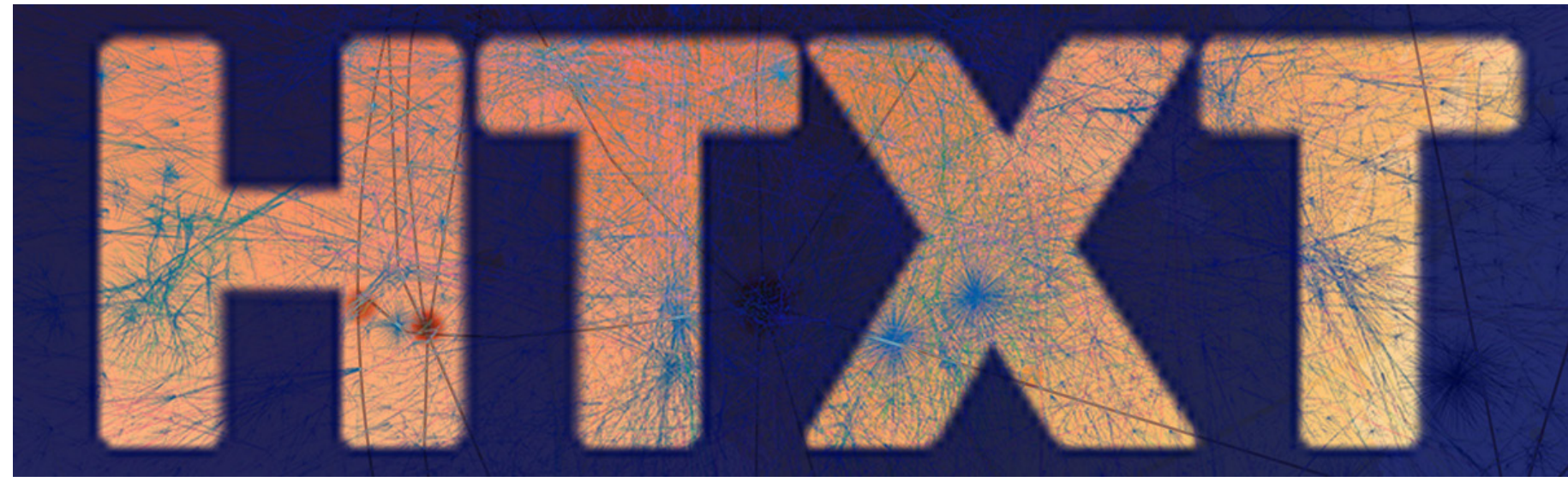

HIPERTEXT.NET 19 | NÚMERO MONOGRÁFICO | MONOGRAPHIC NUMBER | NÚMERO MONOGRÀFIC

Ciencia Abierta, visibilidad y difusión de la producción académica Open Science, visibility and dissemination of academic output Ciència Oberta, visibilitat i difusió de la producció acadèmica https://raco.cat/index.php/Hipertext/issue/view/27910

\section{OCM \\ Observatorio de Cibermedios}

https://observatoriocibermedios.upf.edu/

\begin{tabular}{|l|ll} 
upf. & $\begin{array}{l}\text { Universitat } \\
\text { Pompeu Fabra }\end{array}$ & $\begin{array}{l}\text { Departamento } \\
\text { de Comunicación } \\
\text { Barcelona }\end{array}$ \\
Grupo DigiDoc
\end{tabular}

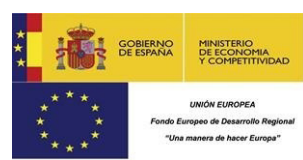

El Observatorio de Cibermedios es una producción del Grupo de Investigación en Documentación Digital y Comunicación Interactiva (DigiDoc) del Departamento de Comunicación de la Universitat Pompeu Fabra.

El Observatorio de Cibermedios (OCM) forma parte del proyecto del Plan Nacional "Creación y contenido interactivo en la comunicación de información audiovisual: audiencias, diseño, sistemas y formatos". CSO2015-64955-C4-2-R (MINECO/ FEDER), Ministerio de Economía y Competitividad (España).

INFORME DIGIDOC - EPI

\section{VISIBILIDAD WEB DE PORTALES DE TELEVISIÓN Y RADIO EN ESPAÑA: ¿QUÉ MEDIOS LLEVAN ACABO UN MEJOR POSICIONAMIENTO EN BUSCADORES?}

